Faculdade de Medicina de Ribeirão Preto

Universidade de São Paulo

Departamento de Clínica Médica

Centro de Ciências das Imagens e Física Médica

ALINE DE ARAÚJO NAVES

"Valor adicional da sequência de difusão na acurácia diagnóstica da ressonância magnética no estadiamento linfonodal de pacientes com neoplasia de próstata"

Ribeirão Preto 


\section{"Valor adicional da sequência de difusão na acurácia diagnóstica da ressonância magnética no estadiamento linfonodal de pacientes com neoplasia de próstata"}

\section{Versão corrigida}

A versão original encontra-se disponível tanto na Biblioteca da Unidade que aloja o Programa, quanto na Biblioteca Digital de Teses e Dissertações da USP (BDTD)

Dissertação apresentada ao Programa de pósgraduação em Ciências das Imagens e Física Médica da Faculdade de Medicina de Ribeirão Preto, como parte das exigências para obtenção do título de Mestre.

Área de Concentração: Diagnóstico por Imagem

Orientador: Valdair Francisco Muglia

Ribeirão Preto 
Autorizo a reprodução e divulgação total ou parcial deste trabalho, por qualquer meio convencional ou eletrônico, para fins de estudo e pesquisa, desde que citada a fonte.

FICHA CATALOGRÁFICA

Naves, Aline de Araújo.

"Valor adicional da sequência de difusão na acurácia diagnóstica da ressonância magnética no estadiamento linfonodal de pacientes com neoplasia de próstata" Naves, Aline de Araújo; Orientador: Muglia, Valdair Francisco Ribeirão Preto, 2017.

35 páginas, 4 gráficos, 4 ilustrações, 8 tabelas.

Dissertação de mestrado (Dissertação apresentada ao programa de pósgraduação em Ciências da Imagens e Física Médica) - Faculdade de Medicina de Ribeirão Preto da Universidade de São Paulo.

1. Sequência de difusão 2. Estadiamento linfonodal. 3. Neoplasia de próstata I. Muglia, Valdair Francisco, orient. II. Título. 
Naves, Aline de Araújo

"Valor adicional da sequência de difusão na acurácia diagnóstica da ressonância magnética no estadiamento linfonodal de pacientes com neoplasia de próstata"

Dissertação apresentada ao Programa de pósgraduação em Ciências das Imagens e Física Médica da Faculdade de Medicina de Ribeirão Preto, como parte das exigências para obtenção do título de Mestre.

Aprovado em:

Banca examinadora:

Prof. Dr.:

Instituição:

Julgamento:

Prof. Dr.:

Instituição:

Julgamento:

Prof. Dr.:

Instituição:

Julgamento: 


\section{AGRADECIMENTOS}

Agradeço aos meus pais pelo apoio irrestrito e amor incondicional.

Aos professores Valdair Muglia e Jorge Elias, pelo exemplo de competência e pelos ensinamentos diários.

À colega Camila Vila Boas, pelo imensa colaboração para a conclusão do trabalho. 


\section{DEDICATÓRIA}

Dedico a todos que sonharam comigo e viveram de perto cada passo dessa etapa tão importante de minha vida acadêmica. 


\section{RESUMO}

Naves, AA. Valor adicional da sequência de difusão na acurácia diagnóstica da ressonância magnética no estadiamento linfonodal de pacientes com neoplasia de próstata [dissertação]. Ribeirão Preto: Universidade de São Paulo; Faculdade de Medicina de Ribeirão Preto; 2017.

INTRODUÇÃO: O adenocarcinoma de próstata (CaP) é a segunda neoplasia maligna de órgãos sólidos mais frequente em homens no mundo. As metástases linfonodais alteram o estadiamento e o prognóstico dos pacientes. objetivo deste trabalho é avaliar a acurácia diagnóstica da Ressonância Magnética de próstata (RMP) na detecção de metástases linfonodais pélvicas e avaliar a acurácia dos critérios morfológicos, da imagem ponderada em difusão (DWI) e coeficiente de difusão aparente (ADC). MATERIAIS E MÉTODOS: No período de 1 de janeiro de 2015 a dezembro de 2016 foram avaliados, por dois observadores, 60 exames de RMP de pacientes que realizaram prostatectomia radical e linfadenectomia pélvica. Em momentos diferentes foi realizada análise morfológica (sequência ponderadas T2) e da DWI, com a medida do ADC e comparado o desempenho de ambas. RESULTADOS: Na análise por paciente da sequência T2 e DWI, a RMP apresentou uma sensibilidade variando de $45,5 \%$ a $63,6 \%$ e especificidade de $87,0 \%$ a $97,6 \%$, sem diferença estatisticamente significativa. Já na análise por lesão os valores da sensibilidade variaram de $15,4 \%$ a $53,8 \%$ e a especificidade de $86,3 \%$ a $90,6 \%$. Apenas para o observador 1 (menos experiente) a DWI aumentou a detecção de linfonodos. Não foi verificada diferença estatisticamente significativa entre as dimensões dos linfonodos positivos e negativos ou do ADC. CONCLUSÃO: A DWI não aumentou a acurácia diagnóstica da RMP no estadiamento linfonodal de pacientes com CaP. O critérios isolados de tamanho e o valor do ADC não apresentaram boa acurácia devido a sobreposição entre os valores dos linfonodos normais e patológico.

Palavras-chave: Neoplasias da próstata. Imagem por Ressonância Magnética. Imagem de Difusão por Ressonância Magnética. Metástase linfática. 


\begin{abstract}
Naves, AA. Incremental value of diffusion-weighted imaging in diagnostic accuracy on lymph node staging of prostate cancer by Magnetic Resonance Imaging [dissertation]. Ribeirão Preto: University of São Paulo, Ribeirão Preto Medical School; 2017.
\end{abstract}

INTRODUCTION: Prostate cancer (PC) is the second most common malignancy in men worldwide. Lymph node metastases change the staging and prognosis of these patients. The objective of this study was to evaluate the adittional value of Diffusionweighted magnetic resonance imaging (DWI-MRI) using mean apparent diffusion coefficient $(A D C)$ to lymph node staging of patients with PC. MATERIALS AND METHODS: Between January 2015 and December 2016, two observers evaluated 60 consecutive MRI exams of patients who underwent radical prostatectomy and pelvic lymphadenectomy. At different times, morphological analysis (T2 sequences) and DWI were performed with the ADC measurement and compared the performance of both. RESULTS: In per patient analysis of T2 and DWI images, MRI showed a sensitivity ranging from $45.5 \%$ to $63.6 \%$ and specificity from $87.0 \%$ to $97.6 \%$, with no statistically significant difference. In per lesion analysis, sensitivity ranged from $15.4 \%$ to $53.8 \%$ and specificity from $86.3 \%$ to $90.6 \%$. Only for observer 1 (less experienced) DWI increased lymph node detection. There was no statistically significant difference between dimensions of the positive and negative lymph nodes as well as ADC measurement. CONCLUSION: DWI did not increase the diagnostic accuracy of MRI in the lymph node staging of patients in PC. Mean ADC and lymph node size, alone or in combination, did not showed good accuracy due to the overlap between normal and pathological lymph node findings.

Keywords: Prostate Cancer. Magnetic resonance imaging. Diffusion. Lymphatic metastasis. 


\section{LISTA DE GRÁFICOS}

Gráfico 1 - ADC médio Linfonodos (+) vs (-) OBS $1 \ldots \ldots \ldots \ldots \ldots \ldots \ldots \ldots \ldots . \ldots \ldots$

Gráfico 2 - ADC médio Linfonodos (+) vs (-) OBS 2 .......................... 12

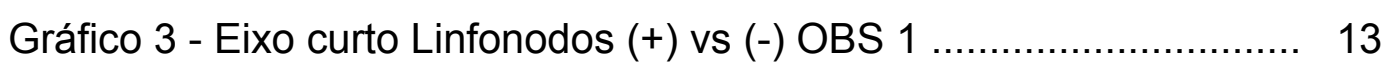

Gráfico 4 - Eixo curto Linfonodos (+) vs (-) OBS 2.......................... 13 


\section{LISTA DE ILUSTRAÇÕES}

Figura 1- Fluxograma da seleção dos pacientes ................................... 05

Figura 2- Caso verdadeiro positivo …………………..................... 14

Figura 3- Caso falso positivo ………………………………....... $\quad 15$

Figura 4- Caso falso negativo ...................................................... 16 


\section{LISTA DE TABELAS}

Tabela 1- Protocolo para a realização da RMP ................................. 06

Tabela 2- Dados clínicos, laboratoriais e patológicos .................... 08

Tabela 3- Análise por paciente. Imagens em T2 e DWI .................. 09

Tabela 4- Análise por lesão. Imagens em T2 e DWI .................... 10

Tabela 5- Valor médio do ADC como critério diagnóstico da linfonodopatia.................................................... 11

Tabela 6- Área sob a curva (AUC) - ADC médio no diagnóstico da linfonodopatia

Tabela 7- Menor eixo no diagnóstico da linfonodopatias

Tabela 8- AUC - Acurácia diagnóstica - avaliada pela AUC - para a medida do menor eixo no diagnóstico da linfonodopatias 


\section{LISTA DE ABREVIATURAS E SIGLAS}

CaP: adenocarcinoma de próstata

PSA: antígeno prostático específico.

RMP: Ressonância Magnética de próstata.

ESUR: European Society of Urogenital Radiology.

DWI: Diffusion weighted imaging - imagem ponderada em difusão

PI-RADS: Prostate Imaging Reporting And Data System

ACR: American College of Radiology

TC: Tomografia Computadorizada

ADC: Apparent diffusion coefficient - coeficiente de difusão aparente

HCFMRP-USP: Hospital das Clínicas da Faculdade de Medicina de Ribeirão Preto, da Universidade de São Paulo

SIH: Sistema de Informação Hospitalar

SIR: Sistema de de laudos radiológicos

PACS: Picture Archiving and Communication System

CCIFM: Centro de Ciência das Imagens e Física Médica

ROC: Receiver Operator Characteristic Curve

VPP: valor preditivo positivo

VPN: valor preditivo negativo

ISUP: International Society of Urological Pathology 
SUMÁRIO

1 INTRODUÇÃO 01

2 OBJETIVOS 03

2.1 Objetivo primário ........................................................ 03

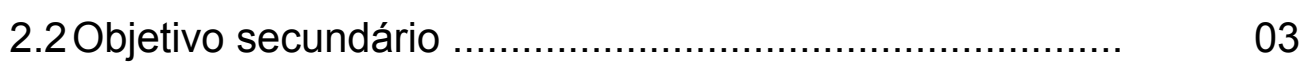

3 METODOLOGIA 04

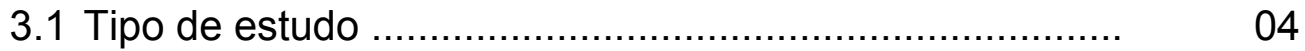

3.2 Local do estudo .......................................................... 04

3.3 Comitê de ética ................................................................

3.4 Coleta de dados ........................................................... 04

3.5 Seleção dos pacientes .................................................... 04

3.5.1 Critérios de inclusão ............................................... 04

3.5.2 Critérios de exclusão ............................................ 05

3.6 Análise dos dados clínicos ........................................... 05

3.7 Análise das imagens .................................................. 06

3.8Análise das peças operatórias ........................................ 07

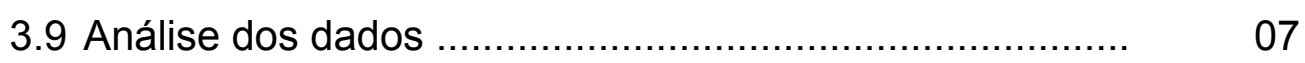

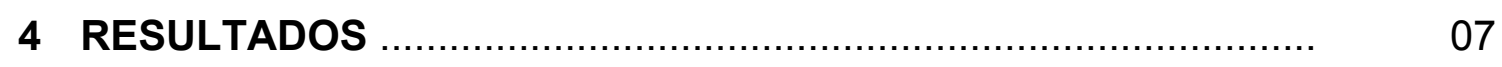

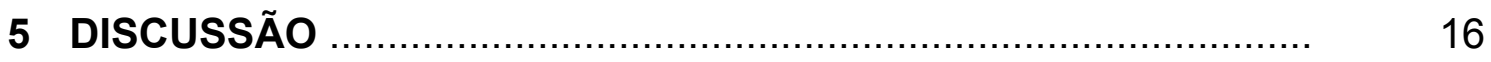

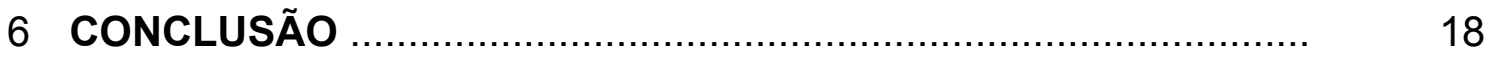

7 REFERÊNCIAS BIBLIOGRÁFICAS ............................................... 19 


\section{INTRODUÇÃO}

O adenocarcinoma de próstata $(\mathrm{CaP})$ é a segunda neoplasia maligna de órgãos sólidos mais frequente em homens no mundo. Estima-se que mais de 3,3 milhões de homens vivam com câncer de próstata nos Estados Unidos e 180.890 casos adicionais foram diagnosticados em 2016 (1). No Brasil, foram estimados 61.200 casos novos deste tipo de câncer em 2016, segundo o Instituto Nacional de Câncer (2).

Quando, no diagnóstico, a doença é restrita à próstata, são grandes as chances curativas com o tratamento local. No entanto, se há o diagnóstico de extensão extraprostática e/ou metástases linfonodais, a taxa de sucesso curativo com o esse tipo de tratamento reduz significativamente, bem como a sobrevida em 5 anos $(3,4)$.

Diversos fatores influenciam a probabilidade de metástases linfonodais. Pacientes com neoplasia prostática confinada ao órgão (estadios T1 e T2) têm uma incidência de metástase linfonodal inferior a $5 \%$, ao passo que, quando há extensão extracapsular a incidência chega a 30\% (5,6). Elevados níveis de antígeno prostático específico (PSA >20 ng/ml) e alto escore de Gleason (>7) na biópsia prostática também aumentam esse risco (6).

Para a interpretação adequada dos métodos de imagem é preciso compreender os padrões de envolvimento linfonodal e suas características. A disseminação do CaP ocorre por via linfática através do feixe neurovascular para as cadeias obturadoras, pré sacral, hipogástricas e ilíacas. As mais acometidas são as obturadoras e ilíacas externas, em $50 \%$ a $60 \%$ dos casos, respectivamente (6).

A Ressonância Magnética de próstata (RMP) tem sido cada vez mais utilizada para o manejo do CaP, especialmente após as recomendações feitas pela European Society of Urogenital Radiology (ESUR) em 2012 (7). Foi proposta a utilização de protocolos com sequências morfológicas, como T2 de alta resolução, e funcionais como a imagem ponderada em difusão (DWl- Diffusion weighted imaging) e o contraste dinâmico (8). Além das recomendações técnicas, a ESUR propôs a padronização dos relatórios com o PI-RADS (Prostate Imaging - Reporting and Data System), que já se encontra em sua segunda versão, juntamente com o American College of Radiology (ACR) (9). 
A RMP contribui na abordagem da neoplasia de próstata, na localização, estadiamento, avaliação da agressividade tumoral e decisão terapêutica (10). A acurácia diagnóstica da RMP para o estadiamento linfonodal do CaP varia muito na literatura, sendo encontrada uma sensibilidade de 6 a $83 \%$ (combinada de 39\%) e uma especificidade de 65 a 99\% (combinada de 82\%) (11). A baixa sensibilidade é explicada pela frequente sobreposição de características morfológicas dos linfonodos pélvicos acometidos por doenças benignas e malignas $(12,13)$.

Um dos parâmetros mais utilizados para essa diferenciação é o tamanho. Oyen et al, no estudo de Tomografia Computadorizada (TC), ao adotarem $6 \mathrm{~mm}$ como limite superior da normalidade para linfonodos pélvicos obtiveram uma sensibilidade de $78 \%$ e uma especificidade de $97 \%$ para o acometimento neoplásico (14). A irregularidade dos contornos tem demonstrado uma acurácia superior a do tamanho para algumas neoplasias, como por exemplo, a de reto (15). Outras modificações da arquitetura interna também podem auxiliar na determinação do envolvimento metastático como a heterogeneidade e a perda da gordura na região central $(16,17)$.

A técnica de difusão por RM (no inglês diffusion-weigthed imaging - DWI) tem a capacidade de fornecer informações funcionais ao quantificar as propriedades de movimento browniano das moléculas de água no tecido. O grau de restrição à difusão de água no tecido biológico é inversamente correlacionado à celularidade do tecido, ao estroma e à integridade das membranas celulares (18). O parâmetro obtido com as imagens por difusão mais utilizado é o coeficiente de difusão aparente (ADC - Apparent diffusion coefficient), que pode ser calculado adquirindo duas ou mais imagens com uma duração e amplitude de gradiente de campo magnético diferentes (valor b), permitindo a construção de um mapa de ADC, portanto resultando em informações quantitativas. Em teoria, quando comparado com tecido normal, o tecido maligno tem maior celularidade e maior relação núcleo/citoplasma, o que pode limitar o movimento aleatório das moléculas de água e, portanto, causar um sinal maior em DWI com um menor ADC $(19,20)$. Em um estudo retrospectivo de linfonodos pélvicos de pacientes com CaP, Eiber et al. verificaram uma diferença significativa entre o valor médio de ADC de linfonodos malignos $\left(1,07 \pm 0,23 \times 10^{-3}\right.$ $\left.\mathrm{mm} / \mathrm{s}^{2}\right)$ e benignos $\left(1,54 \pm 0,25 \times 10^{-3} \mathrm{~mm} / \mathrm{s}^{2}\right)$, tanto nos grupos menores quanto nos maiores de $10 \mathrm{~mm}$. Com um corte de $1,3010^{-3} \mathrm{~mm} / \mathrm{s}^{2}$, obteve-se uma acurácia de $85,6 \%$, com uma sensibilidade de $86,0 \%$ e uma especificidade de $85,3 \%$. A análise 
isolada, pelo tamanho, utilizou o nível de corte de $8 \mathrm{~mm}$ e obteve uma acurácia de $66,1 \%$, sensibilidade de $82,0 \%$ e especificidade de 54,4 (21).

Atualmente, com a maior resolução espacial das imagens por RMP, obtidas com sequência de alta definição, principalmente na ponderação T2, é possível ter informações mais detalhadas do interior dos linfonodos, bem como de seus contornos. Em tese, a combinação dos critérios morfológicos com o dimensional (tamanho) tende a melhorar a acurácia da RMP para caracterização dos linfonodos patológicos. Somando-se a isto, a avaliação pela medida do coeficiente de difusão aparente, é razoável supor que o estadiamento linfonodal dos pacientes com neoplasia de próstata tenha um incremento diagnóstico quando se utiliza uma associação de parâmetros.

Deste modo, considerando-se a escassez de dados sobre o valor da contribuição da técnica de difusão, com a medida do ADC médio, para o estadiamento linfonodal dos pacientes com câncer de próstata, decidimos realizar este estudo retrospectivo, com este propósito.

\section{OBJETIVOS}

\subsection{Objetivo primário}

Avaliar a acurácia diagnóstica da RMP na detecção de metástases linfonodais pélvicas de pacientes com câncer de próstata.

\subsection{Objetivo secundário}

Avaliar acurácia dos critérios morfológicos (tamanho e forma) e do critério de ADC, isoladamente e em conjunto, na detecção de metástases linfonodais no câncer de próstata. 


\section{METODOLOGIA}

\subsection{Tipo do estudo}

Trata-se de um estudo observacional, transversal, retrospectivo realizado em uma única instituição.

\subsection{Local do estudo}

Estudo realizado no Hospital das Clínicas da Faculdade de Medicina de Ribeirão Preto, da Universidade de São Paulo (HCFMRP-USP), através do acesso ao no Sistema de Informação Hospitalar (SIH) e de laudos radiológicos (SIR).

\subsection{Comitê de ética}

O presente trabalho foi encaminhado para o Comitê de Ética do HC-RP com o número 63583416.0.0000.5440, com dispensa do termo de consentimento livre e esclarecido, por se tratar de estudo retrospectivo.

\subsection{Coleta de dados}

Os dados foram coletados a partir dos sistema de informação hospitalar (HIS) para informações clínicas e exames laboratoriais e do sistema de arquivamento de imagens digitais ( do inglês "PACS") do HC-FMRP, onde constam as imagens.

\subsection{Seleção dos pacientes}

Pacientes com diagnóstico de câncer de próstata submetidos a avaliação de ressonância magnética, no pré-operatório de prostatectomia radical e linfadenectomia, no período de janeiro de 2015 à dezembro de 2016.

\subsubsection{Critérios de inclusão}

Pacientes com câncer próstata comprovado histologicamente que realizaram RM de próstata e foram submetidos a prostatectomia radical e linfadenectomia pélvica. 


\subsubsection{Critérios de exclusão}

1. Intervalo entre a realização da ressonância magnética de próstata e o procedimento cirúrgico maior que quatro meses.

2. Protocolo de realização da ressonância magnética de próstata incompleto ou imagens sem qualidade para avaliação.

3. Avaliação histopatológica incompleta.

No período do estudo foram realizadas 434 RMP, destas apenas 197 eram para estadiamento de $\mathrm{CaP}$, sendo que 106 pacientes receberam tratamento não cirúrgico. Dos 63 exames restantes, 3 apresentaram artefatos na DWI, sendo excluídos, totalizando 60 pacientes, com idades entre 46 e 77 anos.

Figura 1- Fluxograma da seleção dos pacientes.

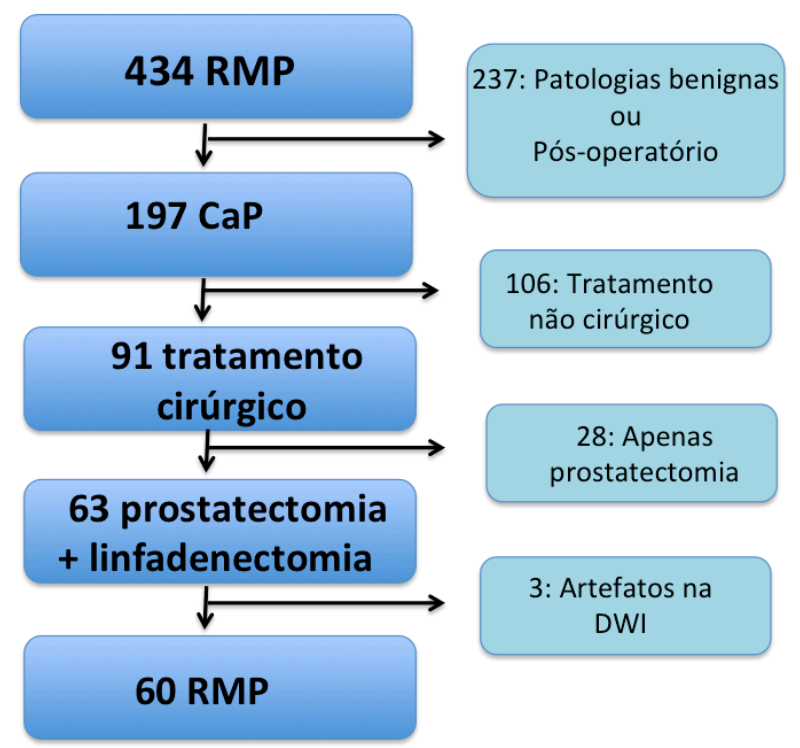

\subsection{Análise dos dados clínicos}

A análise dos dados clínicos foi realizada por um médico observador, residente do terceiro ano de Radiologia e Diagnóstico por Imagem, do Centro de Ciência das Imagens e Física Médica (CCIFM) da HCFMRP-USP e os parâmetros analisados foram: idade, medida do PSA, estadio clínico, data da biópsia, data da realização da RM, data da cirurgia, intervalo entre a RMP e a cirurgia, escore de Gleason da prostatectomia, detalhamento dos achados de linfadenectomia. 


\subsection{Análise das imagens}

Os exames de RMP foram realizados no aparelho Achieva 1,5 Tesla 16 canais-Philips (Best- Netherlands), com o uso de bobina endorretal combinado com bobina pélvica em arranjo de fase ("phased array").

O protocolo realizado baseou-se em cortes com espessura 3-4 $\mathrm{mm}$ de espessura, 0-1 mm de espessamento, FOV de 12 a 16, matriz de 256 × 256 e TE de 120 -160 (Tabela 1). O visualizador utilizado foi o software Horos versão 1.1.7.

Tabela 1 - Protocolo para a realização da RMP

\begin{tabular}{ccccccc}
\hline SEQUÉNCIA & TÉCNICA & $\begin{array}{c}\text { TR/TE } \\
(\mathbf{m s e c})\end{array}$ & $\begin{array}{c}\text { FLIP } \\
\text { (graus) }\end{array}$ & $\begin{array}{c}\text { ESPESSURA } \\
\text { DO CORTE } \\
(\mathbf{m m})\end{array}$ & $\begin{array}{c}\text { FOV } \\
(\mathbf{m m})\end{array}$ & MATRIZ \\
\hline T2 axial & TSE & $3060 / 100$ & 90 & 3 & $150 \times 150$ & $232 \times 184$ \\
T2 coronal & TSE & $2444 / 120$ & 90 & 3 & $150 \times 150$ & $248 \times 198$ \\
T2 sagital & TSE & $3770 / 120$ & 90 & 3 & $260 \times 260$ & $360 \times 275$ \\
DWI axial & SE EPI & $1561 / 71$ & 90 & 5 & $304 \times 375$ & $152 \times 152$ \\
$\begin{array}{c}\text { Perfusão } \\
\text { axial }\end{array}$ & THRIVE & $4 / 2$ & 10 & 4 & $297 \times 345$ & $172 \times 172$ \\
T1 axial & TSE & $443 / 15$ & 90 & 3 & $180 \times 180$ & $180 \times 143$ \\
\hline
\end{tabular}

Legendas: TR = repetition time; TE = echo time; FLIP = flip angle; FOV = field of view; TSE= turbo spin echo; DWI = diffusion-weighted imaging; SE EPI = spin-echo echo-planar imaging; THRIVE = T1 highresolution isotropic volume excitation.

* Os valores de $b$ foram $0,20,500$ e $1000 \mathrm{~s} / \mathrm{mm}^{2}$.

A análise das imagens foi realizada por dois observadores (médicos radiologistas independentes), com quatro e vinte anos de experiência em imagem abdominal, sem acesso às informações clínicas dos pacientes.

Em uma primeira leitura, os seguintes parâmetros morfológicos foram avaliados, imagens das sequências ponderadas em T1 e T2: tamanho, forma e arquitetura interna. Os linfonodos visualizados foram identificados em sua localização e classificados como normais ou patológicos.

A segunda leitura foi realizada dois meses após, analisando o exame completo, incluindo a ponderação em difusão. Todos os linfonodos maiores que 5 
$\mathrm{mm}$ com hipersinal na sequência de DWI foram descritos e classificados como normais ou patológicos. Obteve-se uma medida do ADC médio $\left(10^{-3} \mathrm{~mm}^{2} / \mathrm{s}\right)$ dos linfonodos considerados patológicos e de pelo menos um considerado normal.

\subsection{Análise das peças operatórias}

A análise histopatológica foi realizada pelos dois médicos patologistas da instituição, com 8 e 18 anos de experiência, procurando definir exatamente o local e lado das cadeias linfonodais comprometidas.

\subsection{Análise dos dados}

Todos os gráficos e dados apresentados nesse estudo foram registrados e analisados com o auxílio do programa Stata, versão 14. As variáveis quantitativas foram apresentadas com valores da mediana e média e desvio padrão.

Os dados demográficos foram descritos através da média, mediana e desvio-padrão.

O teste de Shapiro Wilk foi utilizado para verificação da normalidade das variáveis contínuas.

A acurácia diagnóstica foi determinada a partir do cálculo da área sob a curva ROC (receiver operator characteristic curve).

As associações entre cada parâmetro (morfológico e difusão) e a metástase linfonodal foram analisadas através do teste exato de Fisher.

Para todas as comparações adotou-se um nível de significância de 5\% $(p<0,05)$.

\section{RESULTADOS}

O intervalo médio entre a realização da RMP e a cirurgia foi de 2,0 meses. Os pacientes com linfonodos negativos para malignidade apresentaram uma média de idade de 64,3 anos e a mediana do PSA de 37,2 ng/dl. Os com metástase linfonodal possuíam uma média de 59,2 anos e mediana do PSA de 21,3 ng/dl, ambos sem diferença estatisticamente significativa. Estes dados são apresentados na Tabela 2. 
Tabela 2 - Dados clínicos, laboratoriais e patológicos

\begin{tabular}{|c|c|c|c|}
\hline & $\begin{array}{l}\text { Linfonodos negativos } \\
\qquad n=49\end{array}$ & $\begin{array}{l}\text { Linfonodos positivos } \\
\qquad n=11\end{array}$ & $\mathbf{p}$ \\
\hline Idade média (anos) & 64,3 & 59,2 & 0,157 \\
\hline PSA-mediana (ng/dl) & 37,2 & 21,3 & 0,117 \\
\hline \multicolumn{4}{|l|}{ Histopatologia } \\
\hline \multicolumn{4}{|l|}{ (ISUP) } \\
\hline I & $12(24,6 \%)$ & $0(00,0 \%)$ & \\
\hline II & $21(42,8 \%)$ & $3(27,3 \%)$ & 0,010 \\
\hline III & $07(14,3 \%)$ & $4(36,3 \%)$ & \\
\hline IV & $04(08,1 \%)$ & $1(09,1 \%)$ & \\
\hline V & $05(10,2 \%)$ & $3(27,3 \%)$ & \\
\hline \multicolumn{4}{|l|}{$\begin{array}{l}\text { Estadiamento } \\
\text { patológico }\end{array}$} \\
\hline $\mathrm{T} 1 \mathrm{c}$ & $01(02,0 \%)$ & $0(00,0 \%)$ & \\
\hline $\mathrm{T} 2 \mathrm{a}$ & $04(08,1 \%)$ & $0(00,0 \%)$ & 0,023 \\
\hline $\mathrm{T} 2 \mathrm{~b}$ & $03(06,1 \%)$ & $0(00,0 \%)$ & \\
\hline $\mathrm{T} 2 \mathrm{c}$ & $18(36,8 \%)$ & $2(18,2 \%)$ & \\
\hline T3a & $05(10,2 \%)$ & $1(09,1 \%)$ & \\
\hline T3b & $18(36,8 \%)$ & $8(72,7 \%)$ & \\
\hline
\end{tabular}

Dos pacientes com linfonodos negativos, 42,8\% apresentavam estadio histopatológico (ISUP) II, 36.8\% estadio patológico T2c e 36.8\%, T3b. Nenhum dos pacientes com linfonodos positivos era estadio histopatológico I e 72,7\% deles possuíam estadio patológico T3b. Houve diferença estatisticamente significativa para estes parâmetros.

Na análise por paciente (Tabela 3), a avaliação da sequência ponderada em T2 apresentou sensibilidade e especificidade de 54,5\% e 97,6\%, respectivamente para o examinador 1 e de $45,5 \%$ e $63,6 \%$ para o examinador 2. Quando incluída a 
avaliação da DWI, a sensibilidade e especificidade foi de $63,6 \%$ e $87,0 \%$ para o examinador 1 e de $63,6 \%$ e $92,0 \%$ para o 2 . Houve diferença estatisticamente significativa apenas da sensibilidade para o examinador 2.

Tabela 3 - Análise por paciente. Imagens em T2 e DWI

\begin{tabular}{cccccc}
\hline & & Observador 1 & p & Observador 2 & p \\
\hline Sensibilidade & T2 & $54,5 \%(23,4-83,2 \%)$ & 0,31 & $45,5 \%(16,7-76,7 \%)$ & 0,04 \\
& DWI & $63,6 \%(30,8-89,0 \%)$ & & $63,6 \%(30,8-89,1 \%)$ & \\
\hline Especificidade & T2 & $97,6 \%(89,1-99,9 \%)$ & 0,057 & $93,9 \%(83,1-98,7 \%)$ & 0,68 \\
& DWI & $87,0 \%(75,1-94,6 \%)$ & & $92,0 \%(80,8-97,8 \%)$ & \\
\hline VPP & T2 & $85,7 \%(44,5-97,8 \%)^{*}$ & $*$ & $62,5 \%(31,8-85,6 \%)$ & $*$ \\
& DWI & $50,0(30,5-69,5 \%)^{*}$ & & $63,6 \%(38,2-83,2 \%)$ & \\
\hline VPN & T2 & $90,6(83,4-94,8 \%)^{*}$ & $*$ & $88,5 \%(81,6-92,9 \%)$ & $*$ \\
& DW1 & $92,2 \%(84,2-96,3 \%)^{*}$ & & $92,0 \%(83,9 \%-96,2 \%)$ & \\
\hline
\end{tabular}

Apesar da prevalência estimada da doença na amostra ser menor do que a da população, procedeu-se ao cálculo do valor preditivo positivo (VPP), sendo o da análise do T2 de $85,7 \%$ e 50\% com a DWI para o examinador 1. Para o examinador 2, obteve-se VPP de 62,5\% para o T2 e 63,6\% para a DWI.

Para o examinador 1, o valor preditivo negativo (VPN) foi de $90,6 \%$ para o T2 e de 92,2\% quando acrescia a DWI. Para o examinador 2 estes valores foram respectivamente $88,5 \%$ e $92 \%$.

$\mathrm{Na}$ análise por lesão (Tabela 4) do observador 1, obteve-se uma sensibilidade de $15.4 \%$ para o T2 e de $38,5 \%$ para a DWI, com diferença estatisticamente significativa. Já para o observador 2 , a sensibilidade foi de $38,5 \%$ e $53,8 \%$, respectivamente. A especificidade em T2 e DWI foi de 86,3\% e 88,9 \% para o examinador 1 e de $87,1 \%$ e $90,6 \%$ para o examinador 2 , sem diferença estatisticamente significativa. 
Tabela 4 - Análise por lesão. Imagens em T2 e DWI

\begin{tabular}{llllll}
\hline & & Observador 1 & p & Observador 2 & p \\
\hline Sensibilidade & T2 & $15,4 \%(1,9-45,5 \%)$ & 0,001 & $38,5 \%(13,9-68,4 \%)$ & 0,09 \\
& DWI & $38,5 \%(13,9-68,4 \%)$ & & $53,8 \%(25,1-80,8 \%)$ & \\
\hline Especificidade & T2 & $86,3 \%(75,7-93,6 \%)$ & 0,64 & $87,1 \%(76,1-94,3 \%)$ & 0,69 \\
& DWI & $88,9 \%(78,4-95,4 \%)$ & & $90,6 \%(80,7-96,5 \%)$ & \\
\hline VPP & T2 & $18,2 \%(5,1-47,7 \%)^{*}$ & $*$ & $38,5 \%(13,9-68,4 \%)$ & $*$ \\
& DWI & $41,7 \%(21,1-65,6 \%)$ & & $53,8 \%(25,1-80,8 \%)$ & \\
\hline VPN & T2 & $83,8 \%(80,1-86,9 \%)$ & $*$ & $87,5 \%(81,9-91,6 \%)$ & $*$ \\
& DWI & $87,5 \%(81,9-91,6 \%)$ & & $90,8 \%(84,4-94,6 \%)$ & \\
\hline
\end{tabular}

O VPP para o observador 1 foi de 18,2\% para o T2 e 41,7\% para a DWI. Para o 2, os valores foram de respectivamente $38,5 \%$ e $53,8 \%$. O VPP para o observador 1 foi $83,8 \%$ para o T2 e $87,5 \%$ para a DWI, já para o 2 , foi de $87,5 \%$ e $90,8 \%$, respectivamente.

O VPN para o T2 foi de $83,8 \%$ na leitura do T2 e $87,5 \%$ da DWI para o observador 1 e $87,5 \%$ e 90,8\% para o observador 2 .

Como critério diagnóstico, para o observador 1 obteve-se um valor de ADC de $0,928 \times 10^{-3} \mathrm{~mm} / \mathrm{s}^{2}$ para os linfonodos negativos e $0,801 \times 10^{-3} \mathrm{~mm} / \mathrm{s}^{2}$ para os positivos, com diferença estatisticamente significativa.

Para o observador 2, o valor para os linfonodos negativos foi de 0,978 $\times 10^{-3}$ $\mathrm{mm} / \mathrm{s}^{2}$ e de $0,871 \times 10^{-3} \mathrm{~mm} / \mathrm{s}^{2}$ para os positivos, sem diferença estatisticamente significativa (Tabela 5).

A área sob a curva (AUC) para acurácia diagnóstica do ADC médio na detecção da linfonodopatias foi de 0,779 para o observador 1 e de 0,658 para o observador 2. Estes valores são demonstrados na tabela 6, gráficos 1 e 2 . 
Tabela 5 - Valor médio do ADC como critério diagnóstico da linfonodopatia

\begin{tabular}{l|l|l|l|l}
\hline \multicolumn{2}{l|}{} & Linfonodos (-) & Linfonodos (+) & $\mathbf{p}$ \\
\hline $\begin{array}{l}\text { ADC médio } \\
\left(10^{-3} \mathrm{~mm} / \mathrm{s}^{2}\right)\end{array}$ & Obs 1 & $0,928+/-0,160$ & $0,801+/-0,217$ & 0,004 \\
\cline { 2 - 5 } & Obs 2 & $0,978+/-0,227$ & $0,871+/-0,163$ & 0,11 \\
\hline
\end{tabular}

Tabela 6 - Área sob a curva (AUC) - ADC médio no diagnóstico da linfonodopatia

\begin{tabular}{l|l|l|l}
\hline \multirow{2}{*}{ AUC } & Observador 1 & Observador 2 & $\mathbf{p}$ \\
\cline { 2 - 4 } & $0,779(0,713-0,838)$ & $0,658(0,572-0,744)$ & 0.39 \\
\hline
\end{tabular}

Notas: $A \cup C=$ area under ROC curve. ROC = receiver operating characteristic

Gráfico 1 - ADC médio Linfonodos (+) vs (-) OBS 1

\section{ADC OBS 1}

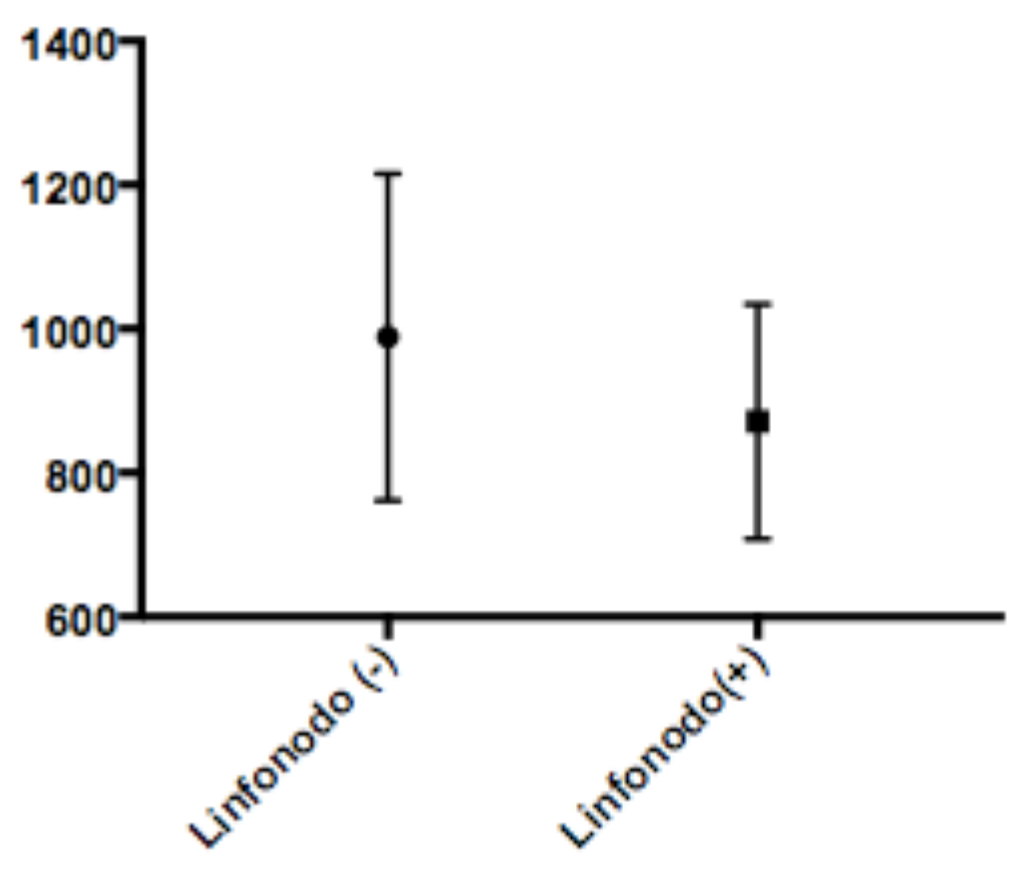

- Linfonodo (-)

- Linfonodo $(+)$ 
Gráfico 2 - ADC médio Linfonodos (+) vs (-) OBS 2

\section{ADC OBS 2}

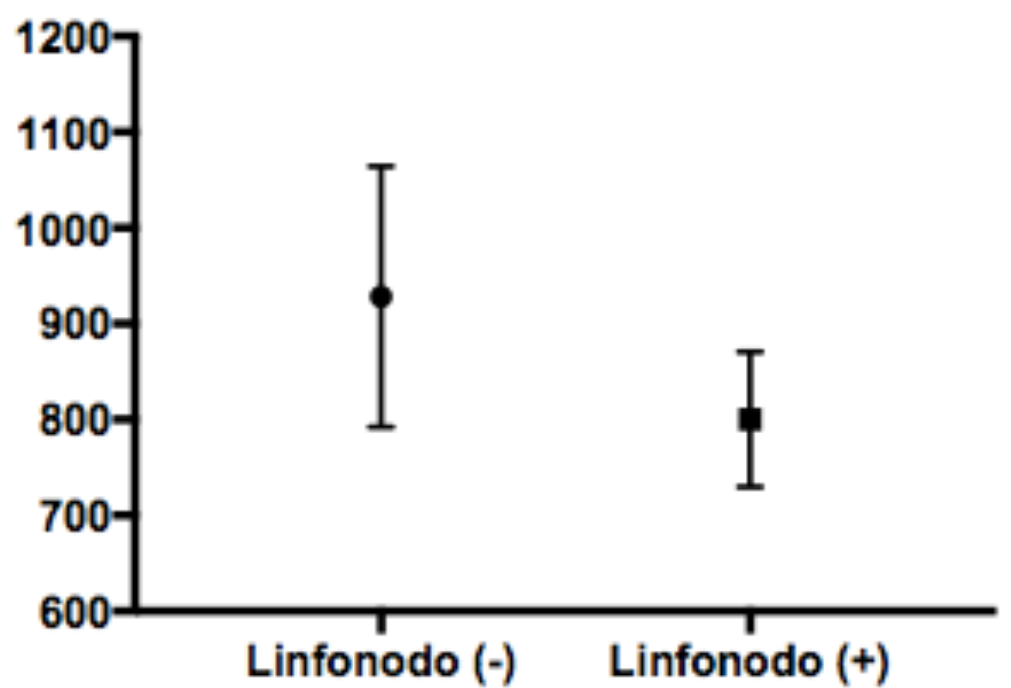

- Linfonodo (-)

- Linfonodo $(+)$

Com relação ao critério diagnóstico do tamanho do eixo curto, para o observador 1 , os negativos mediam, em média $7,1 \mathrm{~mm}$ e os positivos $8,0 \mathrm{~mm}$. Já para o observador 2 , estes valores foram respectivamente $7,1 \mathrm{~mm}$ e $6,7 \mathrm{~mm}$ (Tabela 7).

Tabela 7 - Menor eixo no diagnóstico da linfonodopatia

\begin{tabular}{l|c|c|l|c}
\hline \multicolumn{2}{l|}{} & Linfonodos (-) & Linfonodos (+) & p \\
\hline $\begin{array}{l}\text { Menor } \\
\text { eixo (mm) }\end{array}$ & Obs 1 & $7,10+/-0,19$ & $8,00+/-0,62$ & 0,19 \\
\cline { 2 - 5 } & Obs 2 & $7,12+/-0,25$ & $6,72+/-0,35$ & 0,37 \\
\hline
\end{tabular}

A área sob a curva para a medida do eixo curto foi de 0,648 para o observador 1 e de 0,499 para o 2 . Estes valores estão demonstrados na tabela 8 , gráficos 3 e 4 . 
Tabela 8 - Acurácia diagnóstica - avaliada pela AUC - para a medida do menor eixo no diagnóstico da linfonodopatias

\begin{tabular}{c|c|c|c}
\hline & Observador 1 & Observador 2 & $\mathbf{p}$ \\
\hline AUC & 0,648 & 0,499 & 0,13 \\
& $(0,575-0,724)$ & $(0,421-0,563)$ & \\
\hline
\end{tabular}

Gráfico 3 - Eixo curto Linfonodos (+) vs (-) OBS 1

\section{Eixo curto OBS 1}

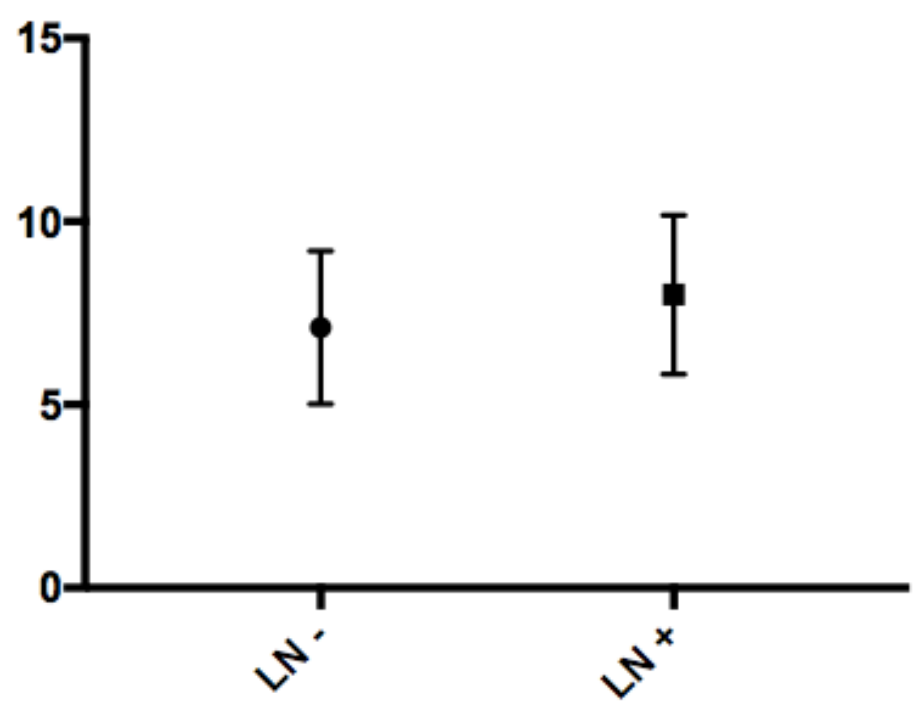

Gráfico 4 - Eixo curto Linfonodos (+) vs (-) OBS 2

\section{Eixo curto OBS 2}

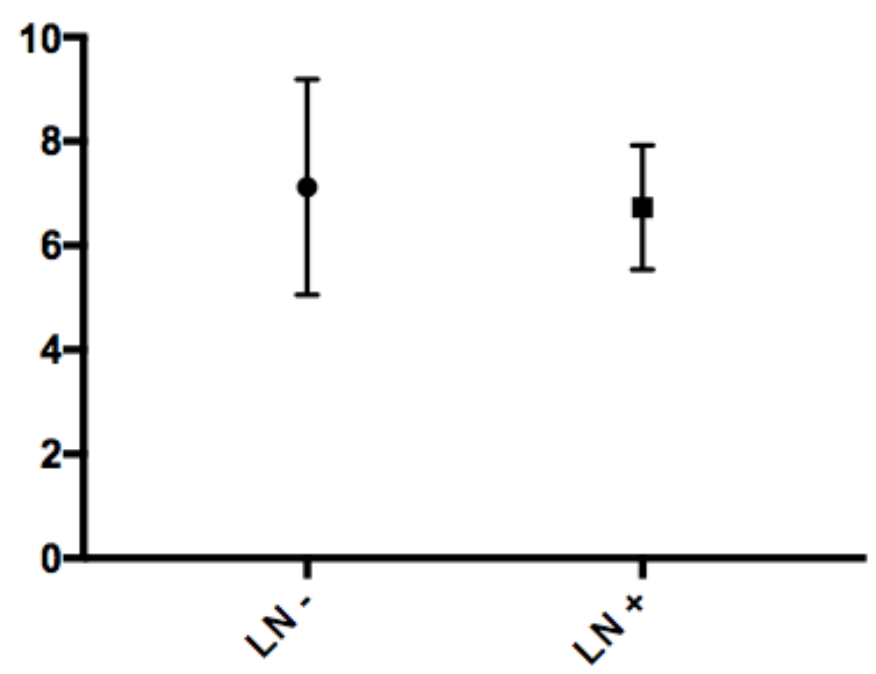


Figura 2 - Caso verdadeiro positivo
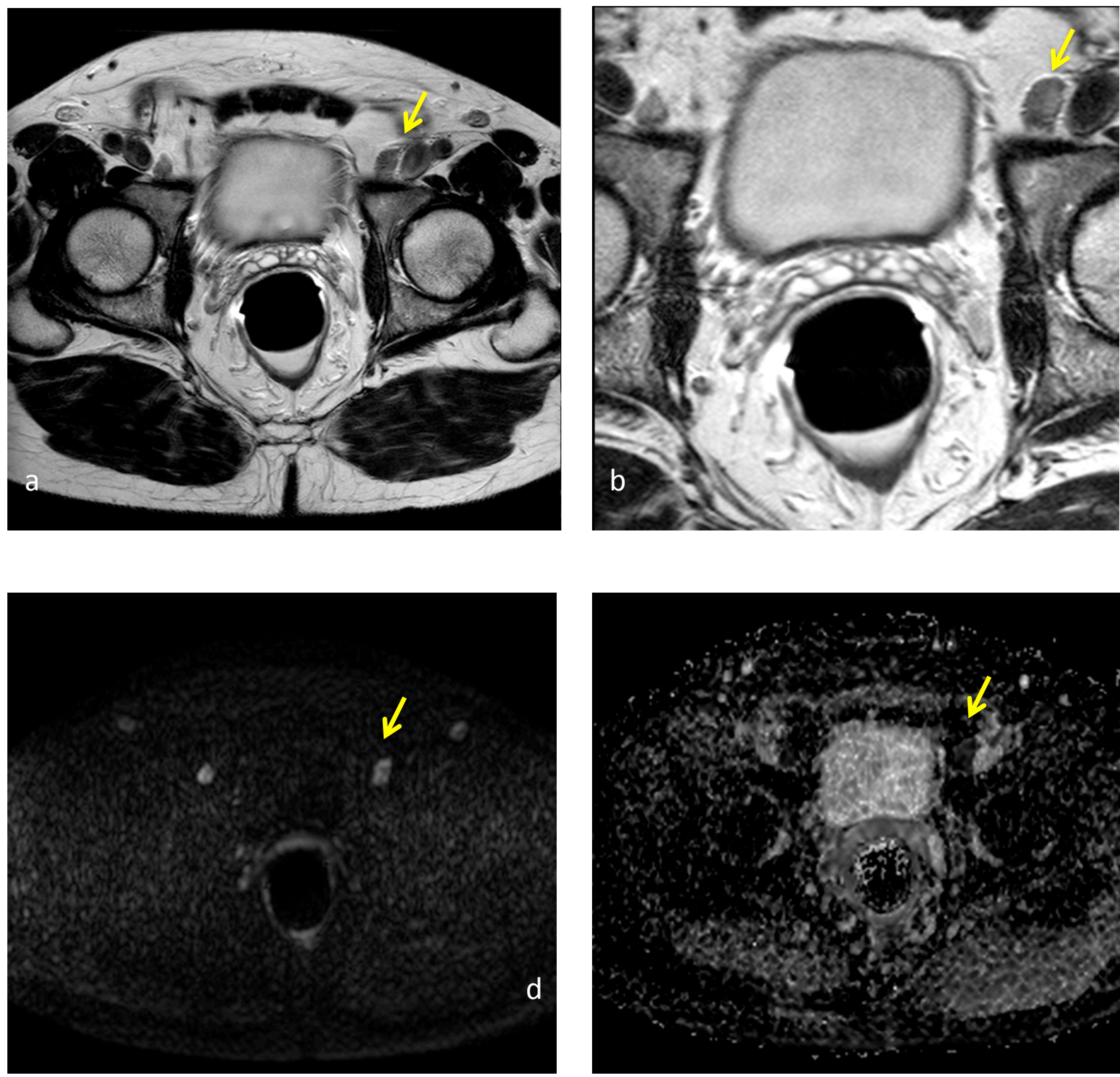

Legenda: Homem de 55 anos, CaP Gleason 9 (5+ 4), apresenta linfonodo de dimensões aumentadas, medindo $10 \mathrm{~mm}$ no eixo curto, localizado na cadeia inguinal esquerda, de contornos irregulares, hipossinal em T2 e perda do hilo gorduroso, mostrado no T2 axial da pelve (a) e T2 axial da próstata (b). O linfonodo apresenta restrição à difusão, com elevado sinal em $\mathrm{DWI}(\mathrm{c})$ e baixo valor de $A D C$, de $0,739 \times 10^{-3} \mathrm{~mm} / \mathrm{s}^{2}$ (d). 
Figura 3 - Caso falso positivo
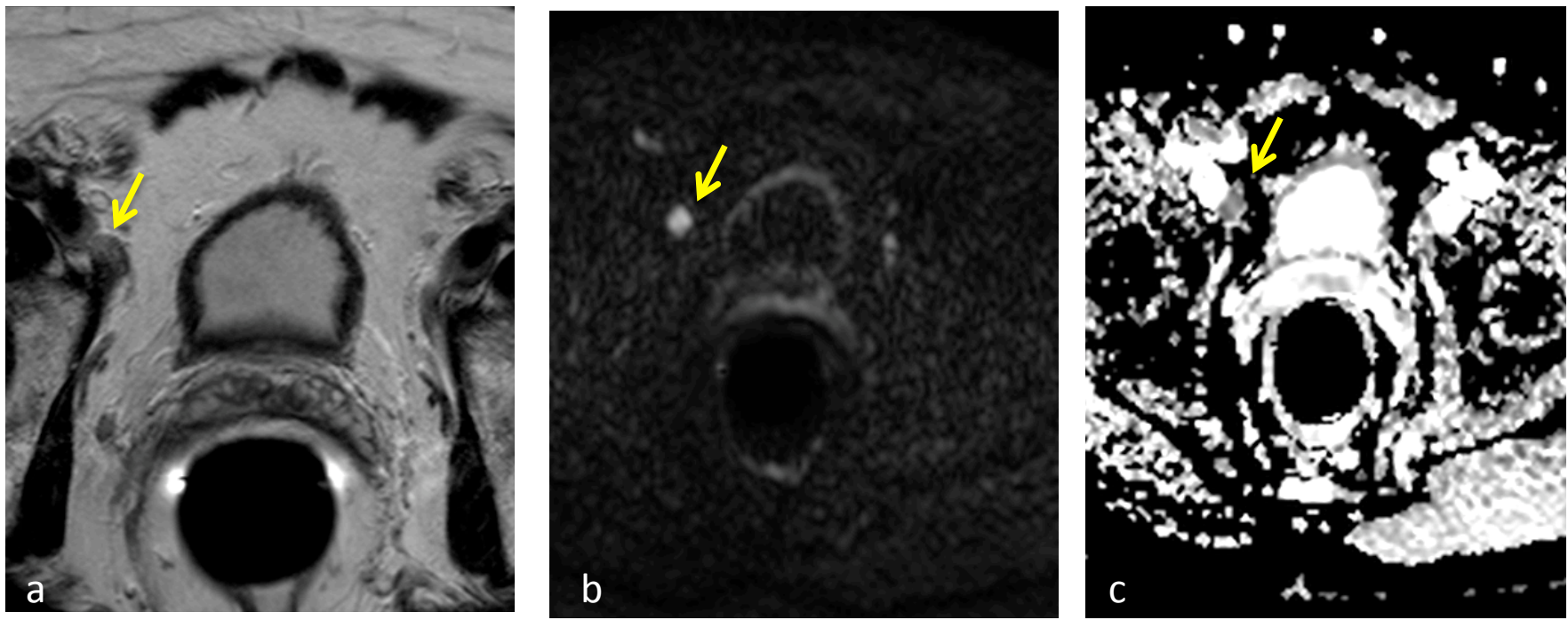

Legenda: Homem de 55 anos, CaP Gleason $7(3+4)$. Na cadeia ilíaca externa direita foi evidenciado linfonodo globoso medindo $6 \mathrm{~mm}$ no eixo curto, com perda da arquitetura interna, mostrado no axial T2 (b). Houve restrição à difusão, com hipersinal em DWI (b) e baixo valor de ADC, de 0,846 x 10-3 $\mathrm{mm} / \mathrm{s}^{2}$ (c).

Figura 4 - Caso falso negativo
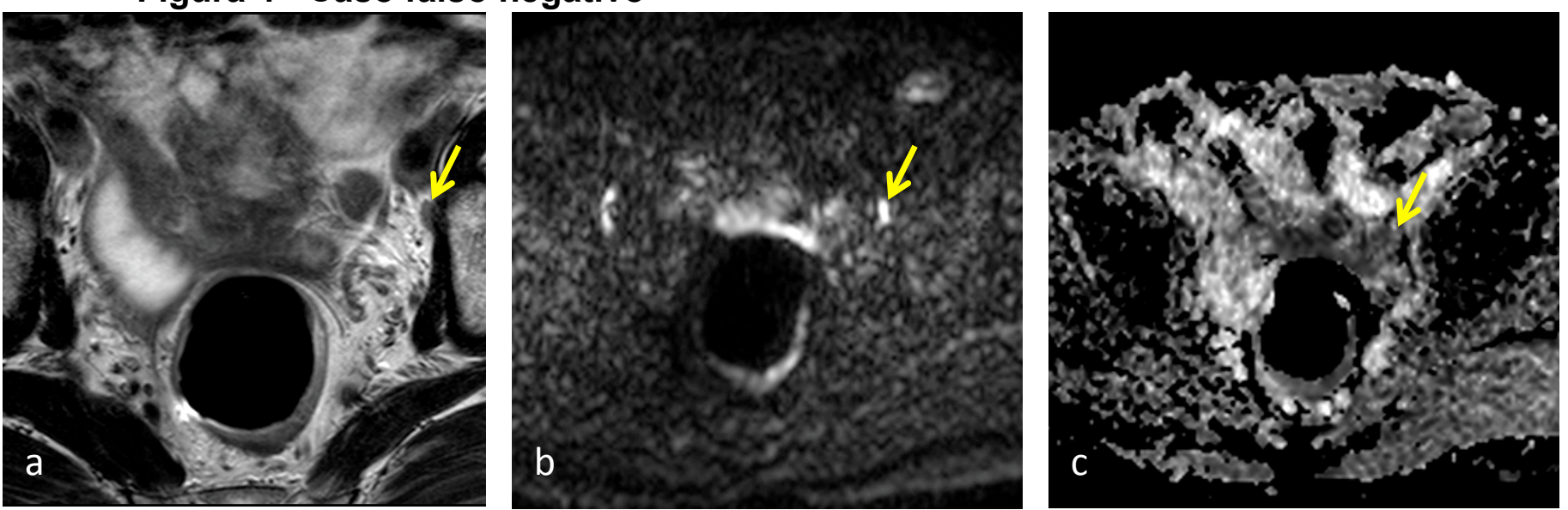

Legenda: Homem de 70 anos, CaP Gleason $9(5+4)$, apresentava linfonodo medindo $4 \mathrm{~mm}$ no eixo curto. Nenhum dos observadores considerou como patológico, o linfonodo localizado na cadeia ilíaca externa esquerda, adjacente ao osso ilíaco, com hipossinal em T2 (b), restrição à difusão, com elevado sinal em DWI (b) e baixo ADC, de $0,843 \times 10^{-3} \mathrm{~mm} / \mathrm{s}^{2}$ (c). 


\section{DISCUSSÃO}

Nossos resultados sugerem que a acurácia da RMP no estadiamento linfonodal de pacientes com neoplasia de próstata é baixa, o que está de acordo valores relatados na literatura (22-25). Além disto, a adição da sequência de DWI, e mesmo o critério isolado de tamanho e a medida do $A D C$, não aumentaram a acurácia do método.

Embora seja esperado um menor PSA nos pacientes sem metástases linfonodais (26), foram encontrados valores similares em ambos os grupos. Isto se justifica pelo fato de que os critérios de elegibilidade para a realização da linfadenectomia baseiam-se em nomogramas que estimam o risco de metástase, e que incluem, dentre outros, elevado valor do PSA e do score de Gleason $(27,28)$. Deste modo, esta ausência de diferença significativa era esperada.

O grau I da ISUP (International Society of Urological Pathology) compreende as neoplasias até Gleason 6 (29,30), que representam lesões de baixa agressividade. Em nosso trabalho, não houve nenhuma metástase linfonodal para este grau. Confirmando a diferença estatisticamente significativa, observou-se que $81 \%$ dos pacientes com linfonodos metastáticos apresentavam estadiamento patológico T3. Este dado está de acordo com diversos trabalhos que demonstraram a relação entre o envolvimento linfonodal e neoplasia prostática avançada $(5,6,31)$.

$\mathrm{Na}$ análise por paciente, das sequências T2 e DWI, a RMP apresentou uma sensibilidade variando de $45,5 \%$ a $63,6 \%$ e especificidade de $87,0 \%$ a $97,6 \%$. Já na análise por lesão, os valores de sensibilidade variaram de $15,4 \%$ a $53,8 \%$ e de especificidade de $86,3 \%$ a $90,6 \%$.

Valores similares são encontrados em outros trabalhos como o de Borley et al, que encontraram, em sua casuística de 42 pacientes com $\mathrm{CaP}$ de alto risco, uma sensibilidade da RMP de $27,3 \%$ e uma especificidade de $100 \%$ para a detecção de metástases linfonodais (22).

Em um estudo prospectivo, Below et al avaliaram 40 pacientes com $\mathrm{CaP}$ de risco intermediário e alto em aparelho de $3 T$, utilizando a $D W I, A D C$, critérios morfológicos e intensidade de sinal em T2 dos linfonodos maiores que $6 \mathrm{~mm}$. Obtiveram uma sensibilidade de 55\%, especificidade de $90 \%$ e acurácia de $72.5 \%$ por paciente. Já para as regiões anatômicas os valores foram respectivamente de 
$41 \%, 94 \%$ e $80 \%(23)$.

Ao avaliar apenas o mapa de ADC de 33 pacientes com CaP, Heck at al obtiveram uma sensibilidade de $57 \%$ e especificidade de $79 \%$ (24). Essa baixa especificidade se deve ao fato dessa sequência apresentar baixa resolução espacial e fornecer menos informações morfológicas (24).

Em nosso estudo, os critérios para a suspeição de malignidade basearam-se nos usados em trabalhos semelhantes prévios. Com relação ao tamanho, tem-se utilizado medidas entre 5 e $15 \mathrm{~mm}$ no menor eixo, sendo que o limite da maioria dos estudos é de 8-10 $\mathrm{mm}$. Jager at al recomendaram o valor de corte $10 \mathrm{~mm}$ para os linfonodos ovalados e $8 \mathrm{~mm}$ para os redondos (16). Neste trabalho não foram avaliados todos os linfonodos, mas apenas aqueles maiores que $5 \mathrm{~mm}$.

Com relação ao tamanho, não verificamos diferença estatisticamente significativa entre as dimensões dos linfonodos positivos e negativos para ambos os observadores. Como o menor eixo utilizado para o diagnóstico foi de $6,5 \mathrm{~mm}$ para o observador 1 e de $5,0 \mathrm{~mm}$ para o 2, reforçamos que o critério isolado do tamanho não apresenta boa acurácia, visto que linfonodos pequenos podem ser sítios de metástases microscópicas (16). Diversos trabalhos enfatizam a necessidade da associação de outros parâmetros de suspeição como a forma globosa, irregularidade dos contornos, alteração da arquitetura interna, como perda do hilo gorduroso e intensidade de sinal heterogênea (15-17,32-34).

A avaliação da DWI foi feita tanto para contribuir na detecção de linfonodos, não visualizados previamente nas imagens ponderadas em T2, quanto para a caracterização, através da mensuração da restrição da difusão, pelo valor do ADC. Apesar de ter havido diferença estatisticamente significativa na detecção do examinador 1, houve muita sobreposição entre os valores do ADC para os linfonodos metastáticos e os normais para ambos os observadores.

A DWI demonstrou contribuir para o aumento da sensibilidade na análise por paciente do observador 1 e na análise por lesão do observador 2. Trabalhos similares mostram que, embora a medida isolada do ADC não demonstre boa acurácia na detecção de pequenas metástases linfonodais $(25,35)$, o uso combinado da DWI com o T2 pode contribuir para a identificação dos linfonodos pélvicos $(35,36)$. 
O presente estudo tem algumas limitações. Sua natureza retrospectiva, o torna mais suscetível a vieses, principalmente o de seleção. O tamanho pequeno da amostra se justifica pela pequena parcela de pacientes que são submetidos à linfadenectomia. E, mesmo quando é realizada, a linfadenectomia estendida, não é possível garantir que todos os linfonodos avaliados pela imagem foram retirados e analisados histologicamente. Optamos por analisar apenas os linfonodos maiores que 5,0 mm devido ao grande número de linfonodos pélvicos, uma vez que a análise de todos levaria a um número elevado de linfonodos, a maioria normal, o que poderia melhorar a acurácia diagnóstica, artificialmente. Além disso, apesar de não ser possível excluir a presença de comprometimento linfonodal em linfonodos com menor eixo inferior a $5,0 \mathrm{~mm}$, a ocorrência deste fato é bastante improvável. Por último, apesar da descrição cirúrgica e da peça patológica ser extremamente detalhada, dada a natureza retrospectiva do estudo, não é possível assegurar uma correlação perfeita entre os achados anatomopatológicos e os de imagem.

\section{CONCLUSÃO}

A imagem ponderada em difusão não aumentou a acurácia diagnóstica da ressonância magnética no estadiamento linfonodal de pacientes com neoplasia de próstata.

O critério isolado do tamanho, bem como o valor do ADC não apresentaram boa acurácia devido a sobreposição entre os valores dos linfonodos normais e patológicos. 


\section{REFERÊNCIAS}

1. Miller KD, Siegel RL, Lin CC, Mariotto AB, Kramer JL, Rowland JH, et al. Cancer treatment and survivorship statistics, 2016. CA Cancer J Clin [Internet]. 2016;66(4):271-89. Available from:

http://doi.wiley.com/10.3322/caac.21149\%5Cnhttp://www.ncbi.nlm.nih.gov/pub $\mathrm{med} / 27253694$

2. Instituto Nacional de Cancer José Alencar Gomes da Silva. INCA - Instituto Nacional de Câncer - Estimativa 2016 [Internet]. Ministério da Saúde Instituto Nacional de Cancer José Alencar Gomes da Silva. 2016. 124 p. Available from: http://www.inca.gov.br/estimativa/2014/sintese-de-resultadoscomentarios.asp

3. Partin AW, Mangold LA, Lamm DM, Walsh PC, Epstein JI, Pearson JD. Contemporary update of prostate cancer staging nomograms (Partin Tables) for the new millennium. Urology. 2001;58(6):843-8.

4. Bluestein DL, Bostwick DG, Bergstralh EJ, Oesterling JE. Eliminating the need for bilateral pelvic lymphadenectomy in select patients with prostate cancer. $J$ Urol. 1994;151(5):1315-20.

5. Bundrick WS, Culkin DJ, Mata JA, Zitman RI, Venable DD. Evaluation of the current incidence of nodal metastasis from prostate cancer. J Surg Oncol. 1993;52(4):269-71.

6. Koh DM, Hughes M, Husband JE. Cross-sectional imaging of nodal metastases in the abdomen and pelvis. Abdom Imaging. 2006;31(6):632-43.

7. Barentsz JO, Richenberg J, Clements R, Choyke P, Verma S, Villeirs G, et al. ESUR prostate MR guidelines 2012. Eur Radiol. 2012;22(4):746-57.

8. Turkbey B, Brown AM, Sankineni S, Wood BJ, Pinto PA, Choyke PL. Multiparametric Prostate Magnetic Resonance Imaging in the Evaluation of Prostate Cancer. CA Cancer J Clin. 2016;66:326-36.

9. Weinreb JC, Barentsz JO, Choyke PL, Cornud F, Haider MA, Macura KJ, et al. PI-RADS Prostate Imaging - Reporting and Data System: 2015, Version 2. Eur Urol. 2016;69(1):16-40.

10. Lin WC, Westphalen AC, Silva GE, Chodraui Filho S, Reis RB, Muglia VF. Comparison of PI-RADS 2, ADC histogram-derived parameters, and their combination for the diagnosis of peripheral zone prostate cancer. Abdom Radiol [Internet]. 2016; Available from:

http://link.springer.com/10.1007/s00261-016-0826-4

11. Hovels AM, Heesakkers RAM, Adang EM, Jager GJ, Strum S, Hoogeveen YL, et al. The diagnostic accuracy of CT and MRI in the staging of pelvic lymph nodes in patients with prostate cancer: a meta-analysis. Clin Radiol. 2008;63(4):387-95.

12. Kotanagi H, Fukuoka T, Shibata Y, Yoshioka T, Aizawa O, Saito Y, et al. The size of regional lymph nodes does not correlate with the presence or absence 
of metastasis in lymph nodes in rectal cancer. J Surg Oncol. 1993;54(4):252-4.

13. Schall JD. aa. Curr Opin Neurobiol [Internet]. 2013;23:269-74. Available from: http://www.ncbi.nlm.nih.gov/pubmed/23246279

14. Oyen RH, Van Poppel HP, Ameye FE, Van de Voorde WA, Baert AL, Baert L $\mathrm{V}$. Lymph node staging of localized prostatic carcinoma with CT and CT-guided fine-needle aspiration biopsy: prospective study of 285 patients. Radiology. 1994;190(2):315-22.

15. Brown G, Richards CJ, Bourne MW, Newcombe RG, Radcliffe AG, Dallimore NS, et al. Morphologic predictors of lymph node status in rectal cancer with use of high-spatial-resolution MR imaging with histopathologic comparison. Radiology. 2003;227(2):371-7.

16. Jager GJ, Barentsz JO, Oosterhof GO, Witjes JA, Ruijs SJ. Pelvic adenopathy in prostatic and urinary bladder carcinoma: MR imaging with a threedimensional TI-weighted magnetization-prepared-rapid gradient-echo sequence. AJR Am J Roentgenol. 1996;167:1503-7.

17. Kim JH, Beets GL, Kim MJ, Kessels AGH, Beets-Tan RGH. High-resolution MR imaging for nodal staging in rectal cancer: Are there any criteria in addition to the size? Eur J Radiol. 2004;52(1):78-83.

18. Hambrock T, Somford DM, Huisman HJ, van Oort IM, Witjes JA, Hulsbergenvan de Kaa $\mathrm{C}$ a, et al. Relationship between apparent diffusion coefficients at 3.0-T MR imaging and Gleason grade in peripheral zone prostate cancer. Radiology. 2011;259(2):453-61.

19. Lee MC, Tsai HY, Chuang KS, Liu CK, Chen MK. Prediction of nodal metastasis in head and neck cancer using a 3T MRI ADC MAP. Am J Neuroradiol. 2013;34(4):864-9.

20. Kim CK, Park SY, Park JJ, Park BK. Diffusion-weighted MRI as a predictor of extracapsular extension in prostate cancer. Am J Roentgenol. 2014;202(3):270-6.

21. Eiber M, Beer AJ, Holzapfel K, Tauber R, Ganter C, Weirich G, et al. Preliminary results for characterization of pelvic lymph nodes in patients wwth prostate cancer by diffusion-weighted MR-imaging. Invest Radiol [Internet]. 2010;45(1):15-23. Available from:

http://ovidsp.ovid.com/ovidweb.cgi?T=JS\&PAGE=reference\&D=emed9\&NEWS $=\mathrm{N} \& \mathrm{AN}=2010039641$

22. Borley N, Fabrin K, Sriprasad S, Mondaini N, Thompson P, Muir G, et al. Laparoscopic pelvic lymph node dissection allows significantly more accurate staging in "high-risk" prostate cancer compared to MRI or CT. Scand J Urol Nephrol [Internet]. 2003;37(5):382-6. Available from: http://www.ncbi.nlm.nih.gov/pubmed/14594685

23. Von Below C, Daouacher G, Wassberg C, Grzegorek R, Gestblom C, Sörensen J, et al. Validation of 3 T MRI including diffusion-weighted imaging for nodal staging of newly diagnosed intermediate- and high-risk prostate 
cancer. Clin Radiol. 2016;71(4):328-34.

24. Heck MM, Souvatzoglou M, Retz M, Nawroth R, Kübler H, Maurer T, et al. Prospective comparison of computed tomography, diffusion-weighted magnetic resonance imaging and [(11)C]choline positron emission tomography/computed tomography for preoperative lymph node staging in prostate cancer patients. Eur J Nucl Med Mol Imaging. 2014;41(4):694-701.

25. Ichikawa T, Erturk SM, Motosugi U, Sou H, lino H, Araki T, et al. High-B-value diffusion-weighted MRI in colorectal cancer. Am J Roentgenol. 2006;187(1):181-4.

26. Sankineni S, Brown AM, Fascelli M, Law YM, Pinto PA, Choyke PL, et al. Lymph Node Staging in Prostate Cancer. Vol. 16, Current Urology Reports. 2015. p. 1-9.

27. Godoy G, Von Bodman C, Chade DC, Dillioglugil O, Eastham JA, Fine SW, et al. Pelvic lymph node dissection for prostate cancer: Frequency and distribution of nodal metastases in a contemporary radical prostatectomy series. J Urol. 2012;187(6):2082-6.

28. Hinev Al, Anakievski D, Kolev NH, Hadjiev VI. Validation of nomograms predicting lymph node involvement in patients with prostate cancer undergoing extended pelvic lymph node dissection. Urol Int. 2014;92(3):300-5.

29. Epstein JI, Allsbrook WCJ, Amin MB, Egevad LL. The 2005 International Society of Urological Pathology (ISUP) Consensus Conference on Gleason Grading of Prostatic Carcinoma. [Internet]. Vol. 29, The American journal of surgical pathology. 2005. 1228-1242 p. Available from:

http://www.ncbi.nlm.nih.gov/pubmed/26492179

30. Egevad L, Delahunt B, Srigley JR, Samaratunga H. International Society of Urological Pathology (ISUP) grading of prostate cancer - An ISUP consensus on contemporary grading. APMIS. 2016;124(6):433-5.

31. Bastian PJ, Boorjian SA, Bossi A, Briganti A, Heidenreich A, Freedland SJ, et al. High-risk prostate cancer: From definition to contemporary management. Vol. 61, European Urology. 2012. p. 1096-106.

32. Thoeny HC, Froehlich JM, Triantafyllou M, Huesler J, Bains LJ, Vermathen P, et al. Metastases in Normal-sized Pelvic Lymph Nodes: Detection with Diffusion-weighted MR Imaging. Radiology [Internet]. 2014;273(1):125-35. Available from:

http://www.ncbi.nlm.nih.gov/pubmed/24893049\%5Cnhttp://www.ncbi.nlm.nih.g ov/pubmed/24893049\%5Cnhttp://pubs.rsna.org.ludwig.lub.lu.se/doi/full/10.114 8/radiol.14132921

33. van den Brekel MW, Stel H V, Castelijns JA, Nauta JJ, van der Waal I, Valk J, et al. Cervical lymph node metastasis: assessment of radiologic criteria.

Radiology [Internet]. 1990;177(2):379-84. Available from: http://radiology.rsna.org/content/177/2/379.abstract\%5Cnhttp://www.ncbi.nlm.ni h.gov/pubmed/2217772 
34. Saokar A, Islam T, Jantsch M, Saksena MA, Hahn PF, Harisinghani MG. Detection of lymph nodes in pelvic malignancies with computed tomography and magnetic resonance imaging. Clin Imaging. 2010;34(5):361-6.

35. Roy C, Bierry G, Matau A, Bazille G, Pasquali R. Value of diffusion-weighted imaging to detect small malignant pelvic lymph nodes at $3 \mathrm{~T}$. Eur Radiol. 2010;20(8):1803-11.

36. Mir N, Sohaib SA, Collins D, Koh DM. Fusion of high b-value diffusionweighted and T2-weighted MR images improves identification of lymph nodes in the pelvis. J Med Imaging Radiat Oncol. 2010;54(4):358-64. 